Research Article

\title{
A Morphology Filter-Assisted Extreme-Point Symmetric Mode Decomposition (MF-ESMD) Denoising Method for Bridge Dynamic Deflection Based on Ground-Based Microwave Interferometry
}

\author{
Xianglei Liu $\mathbb{D}$, Mengzhuo Jiang, Ziqi Liu, and Hui Wang $\mathbb{D}$ \\ Key Laboratory for Urban Geomatics of National Administration of Surveying, Mapping and Geoinformation, \\ Beijing Key Laboratory for Architectural Heritage Fine Reconstruction \& Health Monitoring, \\ Beijing University of Civil Engineering and Architecture, 1 Zhanlanguan Road, Beijing 100044, China
}

Correspondence should be addressed to Xianglei Liu; liuxianglei@bucea.edu.cn and Hui Wang; 2108160218005@stu.bucea.edu.cn Received 14 January 2020; Accepted 6 April 2020; Published 22 June 2020

Academic Editor: Shock and Vibration

Copyright (c) 2020 Xianglei Liu et al. This is an open access article distributed under the Creative Commons Attribution License, which permits unrestricted use, distribution, and reproduction in any medium, provided the original work is properly cited.

Bridge dynamic deflection is an important indicator of structure safety detection. Ground-based microwave interferometry is widely used in bridge dynamic deflection monitoring because it has the advantages of noncontact measurement and high precision. However, due to the influences of various factors, there are many noises in the obtained dynamic deflection of bridges obtained by ground-based microwave interferometry. To reduce the impacts of noise for bridge dynamic deflection obtained with ground-based microwave interferometry, this paper proposes a morphology filter-assisted extreme-point symmetric mode decomposition (MF-ESMD) for the signal denoising of bridge dynamic deflection obtained by ground-based microwave interferometry. First, the original bridge dynamic deflection obtained with ground-based microwave interferometry was decomposed to obtain a series of intrinsic mode functions (IMFs) with the ESMD method. Second, the noise-dominant IMFs were removed according to Spearman's rho algorithm, and the other decomposed IMFs were reconstructed as a new signal. Finally, the residual noises in the reconstructed signal were further eliminated using the morphological filter method. The results of both the simulated and on-site experiments showed that the proposed MF-ESMD method had a powerful signal denoising ability.

\section{Introduction}

Bridges have become one of the important elements of modern urban transportation. However, due to a combination of various factors such as age, environment, human behavior, overload, and geological activities, corrosion and cracks may occur on the surfaces of bridges and increase the deterioration of the bridges, which may ultimately lead to reducing the performance the bridges or even sudden collapses [1]. Serious damage to the bridges may result in serious property damage and casualties. Therefore, it is an essential and extremely important task to carry out the structural health monitoring of the bridges. With the advantage of reflecting the overall stiffness of a bridge structure [2], bridge dynamic deflection has become an important indicator for evaluating the stability of a monitored bridge, which is closely related to its bearing capacity and ability to resist dynamic loads such as strong winds and earthquakes [2-5]. By analyzing the dynamic deflection of monitored bridges, the internal force distributions can be obtained, and the weak position of the bridge structures can be further determined [6]. Therefore, it is necessary to ascertain highprecision bridge dynamic deflections of the monitored bridges.

Compared with the traditional techniques of bridge dynamic deflection measurement such as level gauges and displacement meters, the new technology of ground-based microwave interferometry has the great advantages of noncontact displacement measurement, a wide frequency range of responses, submillimetric displacement sensitivity, 
and a quick setup [7-10]. It has been widely used to obtain the dynamic deflection measurements of bridges in recent years [11, 12]. Stabile et al. [8] applied the technique to estimate the fundamental dynamic parameters of the reinforced concrete Musmeci Bridge in Basilicata Region (southern Italy), and the results have been validated by the comparison with the ones obtained applying consolidated techniques using data from accelerometers and tromometers. Zhou et al. [12] obtained the subsidence time series of the East Lake High-tech Bridge through groundbased interferometric radar, and a case study was conducted on the subsidence of the East Lake High-tech Bridge during subway shield tunnel crossing underneath this bridge. However, during the data acquisition of bridge dynamic deflection using ground-based microwave interferometry, various factors such as the surrounding environment, thrust of wind, ground-motion, complex traffic activities, artificial operation, and the equipment itself will inevitably generate noise in the collected bridge dynamic deflection. These factors can cause the loss of precision of the obtained bridge dynamic deflection and further lead to errors in data analysis when evaluating the stability of the monitored bridge $[5,13]$. Therefore, it is of great importance to reduce the influence of noise in the bridge dynamic deflection obtained by groundbased microwave interferometry.

At present, wavelet transform is one of the widely used methods for signal denoising with a good time-frequency localization characteristic [14]. This method can be used to obtain the wavelet coefficients of signals and noises with different properties at each scale [15]. Moreover, with different wavelet threshold criteria, wavelet transform can be used to distinguish different signals with high frequencies and low frequencies. Wavelet transform has proven to be a useful tool for nonstationary signal analysis [16-18]. However, the results of wavelet transform can be affected by the selection of the wavelet basis function and wavelet threshold criteria, which may cause the distortion of a signal in the time domain $[19,20]$. Moreover, wavelet transform is not suitable for nonlinear signals [20], which means it is not suitable for analyzing the dynamic deflection of a monitored bridge because if the monitored bridge were damaged, the obtained time-series displacement would be a nonlinear signal.

Recently, the empirical mode decomposition (EMD) method has been widely applied to analyze nonlinear and nonstationary vibration signals. This method works to process a signal by performing a timed adaptive decomposition operation that can adaptively decompose a nonstationary signal into a series of intrinsic mode functions (IMFs) [20]. The decomposed components contain the local characteristic signals for different time scales of the source signal [21]. The influence of noise in the obtained vibration signals can be reduced by eliminating a certain number of low-order IMFs with relatively high frequencies [22]. However, the mode mixing effect and the rough trend function can bring out noise with different scales in the decomposed IMFs, which may cause a loss of precision for the EMD denoised method [20, 23]. To solve this problem, the ensemble empirical mode decomposition (EEMD) method has been proposed based on the research of the statistical properties of a Gaussian white noise signal, which is an improvement based on the EMD method [21, 24-28]. This method changes the extremum distribution of the original signal by adding white Gaussian noise and further decomposes the original signal to alleviate the mode-mixing effect using the EMD method [21, 24-30]. However, the EEMD method is easily affected by the amplitude of the added noise and the number of ensemble trials, which can bring out some residual noise in the decomposed IMFs. Moreover, although the EMD and EEMD methods can effectively reduce the influence of periodic noise, it is difficult to reduce the influence of instantaneous noise, such as the instantaneous vibrations of the equipment itself caused by passing vehicles [31]. Extreme-point symmetric mode decomposition (ESMD) is a new alternative time-frequency analysis method for the EMD and EEMD methods [32]. The main advantage of this method is the ability to adaptively determine the optimal global average curve. Any complex raw signal can be decomposed into a series of different physical meaning IMFs, which means that the ESMD method is superior to the EMD and the EEMD methods $[7,20,33]$. It has been applied in various fields, such as information science, marine and atmospheric science, and seismology [31-35]. Although the ESMD method can effectively reduce the influence of the mode-mixing problems, it is difficult to reduce the influence of noise with different frequencies and scales in decomposed IMFs using the ESMD method.

A mathematical morphology filter is a very typical nonlinear signal processing and analysis technology that has been rapidly developed in recent years [36-39]. This technology has been widely applied in the analysis of signals such as vibration signals and pulse wave signals [40-42]. Morphological filtering based on the theory of mathematical morphology is a new nonlinear filtering method for eliminating noise while protecting signal details. The theory of mathematical morphology is an integrated theory of information theory, statistical estimation theory, adaptive theory, random noise theory, and other theories [36-39]. Morphological filtering is a noise removal algorithm that is suitable for all types of noise and has a better elimination effect [41]. Compared with traditional linear filtering, morphological filtering is more efficient to a certain extent, and it overcomes the shortcomings of linear filtering in which some primary features of the original signal are harmed or blurred while smoothing the noise. Thus, the main morphological features of each signal remain more intact [29, 36]. Moreover, a morphological filter is more effective in reducing impulse noise and white noise, and it will not cause an abrupt change in the frequency domain or a phase delay in the time domain $[29,43]$. To address modemixing problems, Zhou et al. [29] proposed a morphological filter-assisted ensemble empirical mode decomposition (MF-EEMD) for signal denoising that could significantly mitigate the mode-mixing problems and achieve a higher decomposition efficiency compared to the EEMD method.

Therefore, in this study, to effectively eliminate the influences of noise in the dynamic deflection of the monitored 
bridges obtained by ground-based microwave interferometry, a morphology filter-assisted extreme-point symmetric mode decomposition (MF-ESMD) denoising method for bridge dynamic deflection obtained by ground-based microwave interferometry was proposed. The ESMD method was used to decompose the original obtained bridge dynamic deflection into a series of IMFs, and the noisedominant IMFs were further removed according to Spearman's rho algorithm. A morphological filter was used to further eliminate the residual noises in the reconstructed signal. The MF-ESMD denoising method is introduced in Section 2. Section 3 introduces the results and analysis of the simulation experiments, and Section 4 introduces the results and analysis of the on-site experiment, followed by a conclusion in Section 5.

\section{Methods}

Figure 1 shows the entire workflow of the proposed MFESMD denoising method for the bridge dynamic deflection obtained by ground-based microwave interferometry. It consisted of the following two technologies: (1) the original dynamic deflection signal obtained by ground-based microwave interferometry was decomposed into a series of IMFs using the ESMD method. Spearman's rho of each decomposed IMF was calculated to remove the noisedominant IMFs from the decomposed IMFs. (2) A new signal was reconstructed by the remaining IMFs, and the morphological filter method was used to remove the residual noise from the reconstructed signal.

\subsection{Reduction of Noise-Dominant IMFs. The ESMD method} has a better performance in decomposing a signal into a series of physically meaningful representations compared with the EMD and EEMD methods, which can be more effective to separate noise and useful signals [20]. Moreover, as a nonparametric coefficient correlation analysis method, during the process of the calculation of data correlations, Spearman's rho only depends on ranks, which can guarantee enough robustness of signal noise processing [30, 44]. Therefore, in this study, the ESMD method and Spearman's rho were integrated in order to remove the noise-dominant IMFs from the decomposed IMFs for the bridge dynamic deflection obtained by ground-based microwave interferometry. For the original dynamic deflection signal $y(t)$, the detailed process of the reduction of noise-dominant IMFs is shown as follows:

Step 1: All the maximum and minimum extreme points of the original dynamic deflection signal $y(t)$ were found, and the midpoints of the two adjacent extreme points were defined as $F_{i}(i=1,2, \ldots, n-1)$. The midpoints of the left and right borders were set to $F_{0}$ and $F_{n}$ using a linear interpolation method. Interpolation curves (named $L_{1}, L_{2}, \ldots, L_{m}(m \geq 1)$ ) were curved using cubic spline interpolation based on the $N+1$ points in $F_{i}(i=0,1,2, \ldots, n)$, and their average curve $\bar{L}=\left(L_{1}+L_{2}+\cdots+L_{m}\right) / m$ was further calculated.
Step 2: $y(t)-\bar{L}$ was calculated by repeating Step 1 to obtain $\operatorname{IMF}_{1}$ until $|\bar{L}| \leq \varepsilon$ was satisfied or the sifting times reached the preset maximum value $K_{p}$ of 30 . In this study, the permitted error $\varepsilon$ was set to $0.001 \sigma_{0}$, where $\sigma_{0}$ was the standard deviation of the original data $y(t)$ relative to the total mean of $y(t)$.

Step 3: Steps 1 and 2 were repeated for $y(t)-\mathrm{IMF}_{i}$ to obtain $\mathrm{IMF}_{2}, \ldots, \mathrm{IMF}_{n}$ and a residual $R$, which is an optimal adaptive global mean (AGM) curve with no more than a certain number of extreme points. Then, the variance $\sigma$ of $y(t)-R$ was calculated.

Step 4: The sifting times $K$ was changed on a finite integer interval $\left[K_{\min }, K_{\max }\right]$. Then, Steps $1-3$ were repeated to obtain the optimal sifting time $K_{0}$, according to the minimum variance $\sigma_{\min }$ on $\left[K_{\min }, K_{\max }\right]$.

Step 5: The optimal sifting times value $K_{0}$ and the permitted error $\varepsilon$ were used to perform the optimal signal decomposition for the dynamic deflection signal $y(t)$ to obtain a series of IMFs with an optimal AGM curve.

Step 6: For each decomposed IMF, the noise-dominant IMFs had features that were less similar to the features of the original dynamic deflection signal. Spearman's rho between each decomposed IMF and the original dynamic deflection signal is between $[-1,+1]$. The bigger the coefficient value, the more similar it was to the original signal $[30,44]$. Through the coefficient value, noise-dominant IMFs could be removed. Spearman's rho $s(i)$ between the decomposed $\mathrm{IMF}_{i}$ and the original signal $X(t)$ could be calculated using equation (1):

$$
s(i)=\rho\left(y(t), \operatorname{IMF}_{i}(t)\right)=1-\frac{6 \sum_{i=0}^{N}(y(t)-\operatorname{IMF}(t))^{2}}{\left(N^{2}-1\right) N},
$$

where $\operatorname{IMF}_{i}$ is the $i_{\text {th }} \operatorname{IMF}(i=1,2, \ldots, n)$ and $N$ is the number of elements of the original dynamic deflection signal.

2.2. Reduction of the Residual Noise. After the reduction of the noise-dominant IMFs, the other decomposed IMFs were reconstructed as a new signal. There was only a small amount of residual noise in the reconstructed signal, which may be caused by the surrounding environment, artificial operation, and the equipment itself. A morphology filter is used to perform the reduction of the residual noise in the reconstructed signal. Generally, dilation and erosion are two most basic operations in the algorithm of the mathematical morphology method [14-18]. A was defined as a signal, and $B$ was defined as a structuring element. The dilation operation $A \oplus B$ was defined as shown in equation (2), and the erosion operation $A \Theta B$ was defined as shown in equation (3). Based on the dilation and erosion operations, the opening operation $A \circ B$ was defined as shown in equation 


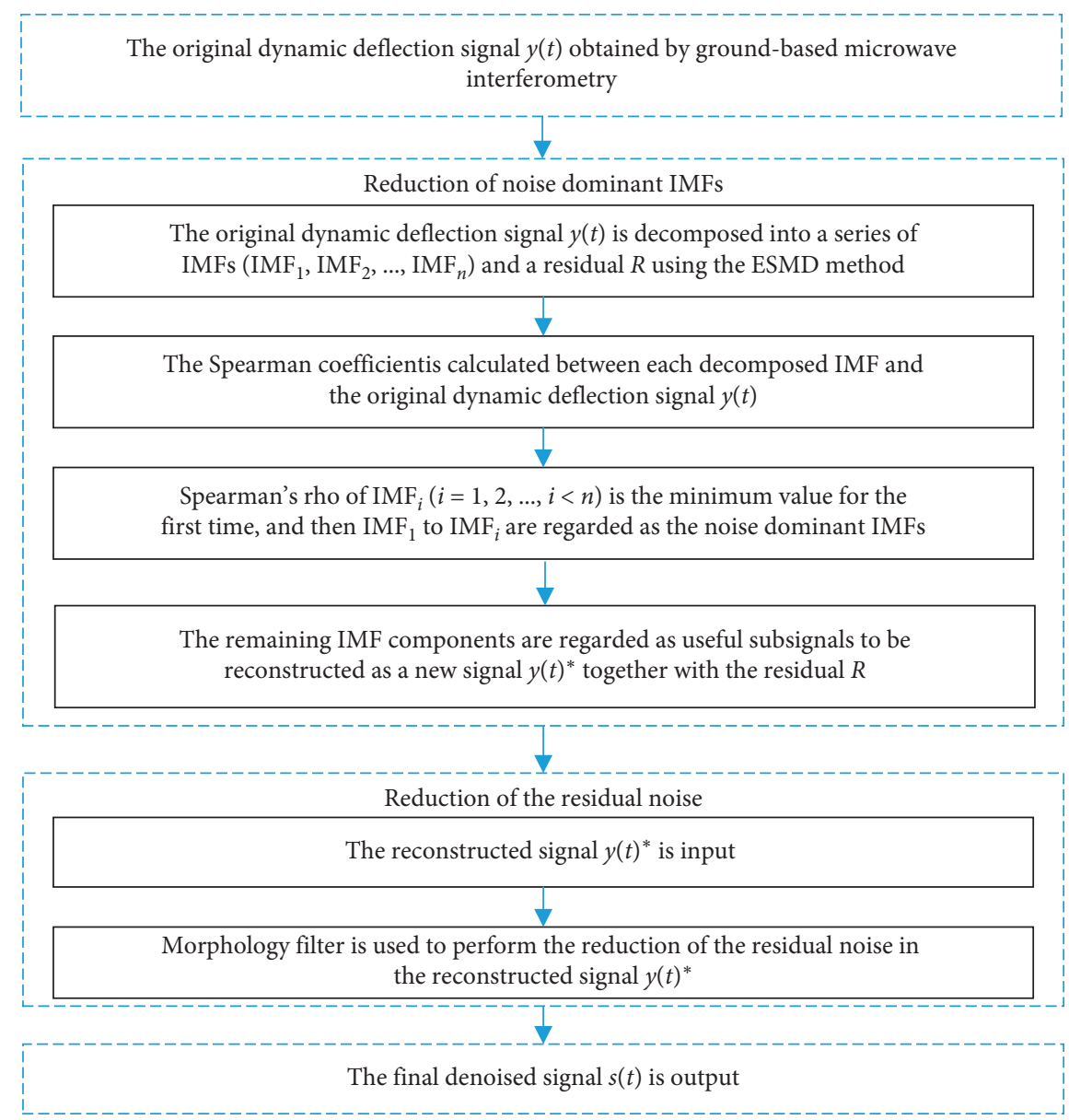

Figure 1: Workflow of the MF-ESMD denoising method.

(4), and the closing operation $A \cdot B$ was defined as shown in equation (5):

$$
\begin{aligned}
& A \oplus B=\max \{A(i-j)+B(j)\}, \\
& A \Theta B=\min \{A(i+j)-B(j)\}, \\
& A \circ B=(A \Theta B) \oplus B, \\
& A \cdot B=(A \oplus B) \Theta B,
\end{aligned}
$$

where $1<i<n, 1<j \leq k, i+j \leq n, n$ is the length of $A$, and $k$ is the width of $B$.

However, the opening operation could only change the maxima and maintain the shape of the minima for the reconstructed signal. The closing operation could only change the minima and maintain the shape of the maxima for the reconstructed signal. Therefore, a good filtering result could not be obtained if any operation was used on its own. However, the average value of the two operations could be very close to the original signal [36]. To address both the minima and maxima of the signal, a morphological filter was proposed in order to reduce the residual noise in the reconstructed signal, as shown in equation (6), which integrated the opening and closing operations:

$$
s(t)=\frac{1}{2}\left[\left(y(t)^{*} \circ g_{1} \bullet g_{2}\right)(n)+\left(y(t)^{*} \bullet g_{1} \circ g_{2}\right)(n)\right],
$$

where $s(t)$ is the final denoised signal, $y(t)^{*}$ is the reconstructed signal, and $n$ is the length of signal $y(t)^{*} \cdot g_{1}$ and $g_{2}$ are the two structuring elements with different sizes, of which the lengths were less than the length of the reconstructed signal $y(t)^{*}$. In addition, the structuring element was an important factor affecting the results of the signal denoising. The most frequently used structuring elements include linear structuring elements, triangular structuring elements, and structuring elements [36-39]. The filtering results were affected by the length and shape of the structuring elements. Therefore, in the actual processing of the signal, the structuring elements were designed according to the characteristics of the analysis signal and the computational complexity [36-39]. In this study, the linear structuring elements were selected due to the simplicity in calculation, and the shape had little effect on the analysis [27].

\section{Simulated Experiments and Analysis}

The dynamic deflection signal of bridges obtained by ground-based microwave interferometry is a complicated 
nonstationary signal mixed with some noise. Therefore, in this study, to validate the feasibility and accuracy of the proposed MF-ESMD denoising method, a nonlinear and nonstationary simulated signal $Y(t)$ was generated that consisted of three basic functions with the different frequencies $X(t)=X_{1}+X_{2}+X_{3}$ and a white Gaussian noise signal with an SNR (signal-to-noise ratio) of $20 \mathrm{~dB}$ to enhance noise information in the simulated signal $Y(t)$, which is shown as follows:

$$
Y(t)=X_{1}+X_{2}+X_{3}+W(t)
$$

where $X_{1}=\cos (20 \pi t+2 \sin (3 \pi t)), \quad X_{2}=\sin (6 \pi t), \quad X_{3}=$ $\cos (10 \pi t)$, and $W(t)$ is a white Gaussian noise signal. The waveforms of the simulation signal $Y(t)$ and its component signals $X_{1}, X_{2}$, and $X_{3}$ are shown in Figure 2 .

As shown in Figure 3, the simulated signal $Y(t)$ was decomposed into eight IMFs with the corresponding meaningful inherent natural frequency from high to low frequency and a residual $R$ using the ESMD method. Then Spearman's rho of each decomposed IMFs was calculated, as shown in Table 1. The value of Spearman's rho from the first decomposed $\mathrm{IMF}_{1}$ was gradually reduced. Spearman's rho of $\mathrm{IMF}_{3}$ was 0.0291 , which was the minimum value for the first time. This indicated that the decomposed IMF 1 to IMF 3 had less similar feathers than the original signal $Y(t)$. Therefore, the first three IMF components, $\mathrm{IMF}_{1}$ to $\mathrm{IMF}_{3}$, were regarded as the noise-dominant IMFs, which may have been caused by the surrounding environment, thrust of wind, ground-motion, complex traffic activities, artificial operation, and the equipment itself. The remaining IMF components were regarded as useful subsignals to be reconstructed as a new signal $Y(t)^{*}$ along with the residual $R$.

For the reconstructed signal $Y(t)^{*}$, most of the obvious noises were reduced. However, there was a small amount of residual noise in the reconstructed signal $Y(t)^{*}$. Therefore, a morphology filter was used to perform the reduction of the residual noise in the reconstructed signal $Y(t)^{*}$. In the morphology filter, $g_{1}=\left[\begin{array}{lll}1 & 1 & 1\end{array}\right]$ and $g_{2}=\left[\begin{array}{lllll}1 & 1 & 1 & 1 & 1\end{array}\right]$ were denoted as shown in equation (6). Then, the final denoised signal $S(t)$ could be obtained using the morphology filter. Figure 4 shows the compared results of the curves between the original signal $X(t)$ and the denoised signal $S(t)$. Moreover, to validate the accuracy and robustness of the proposed MF-ESMD method for signal denoising, morphological filter and MF-EEMD methods $[27,29]$ were selected for comparison with the proposed MFESMD method. The comparison results of the curves between the original signal $X(t)$ and the denoised signal using the morphological filter and the MF-EEMD methods are shown in Figures 5(a) and 5(d). The inspection of the curves from Figures 4 and 5 highlighted the following aspects: (1) for the middle parts of the curve, as shown in Figures 4(b), $5(b)$ and 5(f), the middle parts of the denoised signal were generally consistent with the original signal using the above three methods. The results indicated that the noise could be eliminated effectively using the above three methods for the middle parts of the curve of the simulated signal. (2) For the peak parts of the curve, although there was a maximum deviation of $0.006 \mathrm{~mm}$, the curve of the denoised signal was generally consistent with the original signal using the proposed MF-ESMD method, as shown in Figures 4(c) and 4(d). However, for the denoised signal using the morphological filter and MF-EEMD methods, as shown in Figures 5(c), $5(\mathrm{~d}), 5(\mathrm{~g})$, and $5(\mathrm{~h})$, there was obvious noise in the peak parts of the curve. The results indicated that the proposed MFESMD method had a better performance for peak signal denoising than the morphological filter and the MF-EEMD method.

In order to further evaluate the quality of signal denoising using the proposed MF-ESMD method, two objective evaluation indices, the SNR (signal-to-noise ratio) and RMSE (root-mean-square error), were used in this study. The SNR was a ratio of the useful signal power to the noise power, as shown in equation (8). Generally, the greater the SNR, the better the denoised effect. The SNR could reflect the proportional relationship between the effective part of the original signal and the noise, and it could reflect the denoising ability of the algorithm $[7,45]$. The RMSE was a deviation value between the observed value and the true value, as shown in equation (9). Generally, the smaller the RMSE value, the better the denoised effect. The RMSE could indicate the difference between the original signal and the denoised signal $[7,20]$. The bigger the SNR value, the smaller the RMSE value and the better the effect of noise reduction:

$$
\begin{aligned}
\mathrm{SNR} & =10 \lg \left(\frac{\sum_{n=1}^{N}(s(n))^{2}}{\sum_{n=1}^{N}(y(n)-s(n))^{2}}\right), \\
\mathrm{RMSE} & =\sqrt{\frac{1}{N} \sum_{n=1}^{N}(y(n)-s(n))^{2},}
\end{aligned}
$$

where $y(n)$ is the $n_{\text {th }}$ element of the original signal, $s(n)$ is $n_{\text {th }}$ the element of the denoised signal, and $N$ is the number of elements of the signal.

The comparison results are shown in Table 2. The inspection from this table highlighted the following aspects: (1) the RMSE was $0.0361 \mathrm{~mm}$ for the proposed MF-ESMD denoising method, which was smaller and closer to zero than the RMSE values of the morphological filter method and the MF-EEMD method. (2) The SNR of the simulation signal $Y(t)$ was $20 \mathrm{~dB}$. The SNR was $26.3066 \mathrm{~dB}$ for the denoised signal using the morphological filter method, which increased by $31.53 \%$ compared to the simulation signal $Y(t)$. The SNR was $26.4298 \mathrm{~dB}$ for the denoised signal using the MF-EEMD method, which increased by $32.15 \%$ compared to the simulation signal $Y(t)$. The SNR was $30.6619 \mathrm{~dB}$ for the denoised signal using the proposed MF-ESMD denoising method, which increased by $53.3 \%$ compared to the simulation signal $Y(t)$. The results showed that the proposed MF-ESMD denoising method had a more powerful denoising ability for a nonlinear and nonstationary signal than the morphological filter method and the MF-EEMD method. 


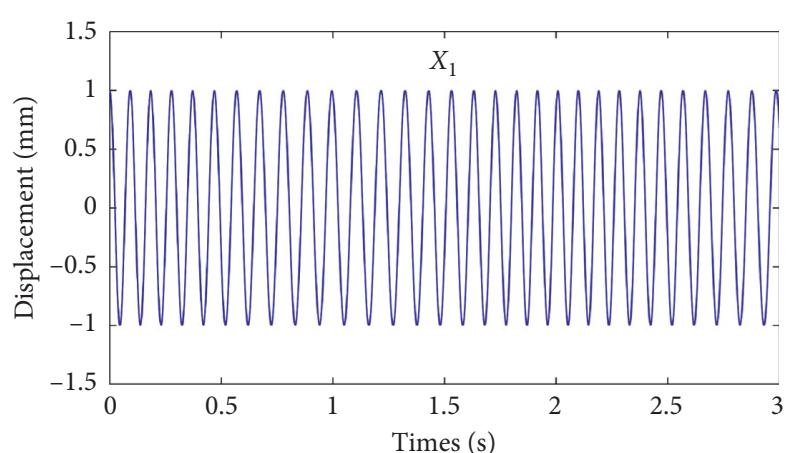

(a)

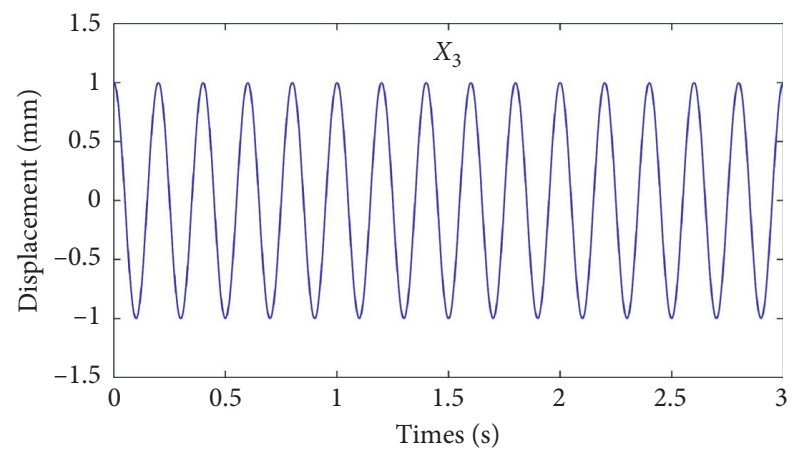

(c)

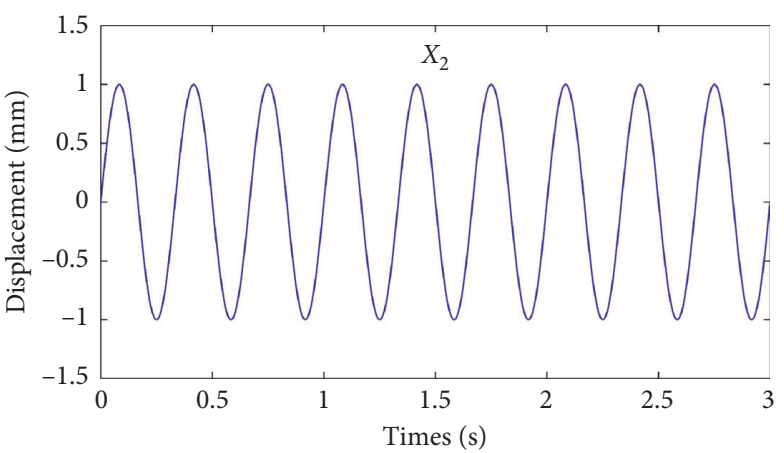

(b)

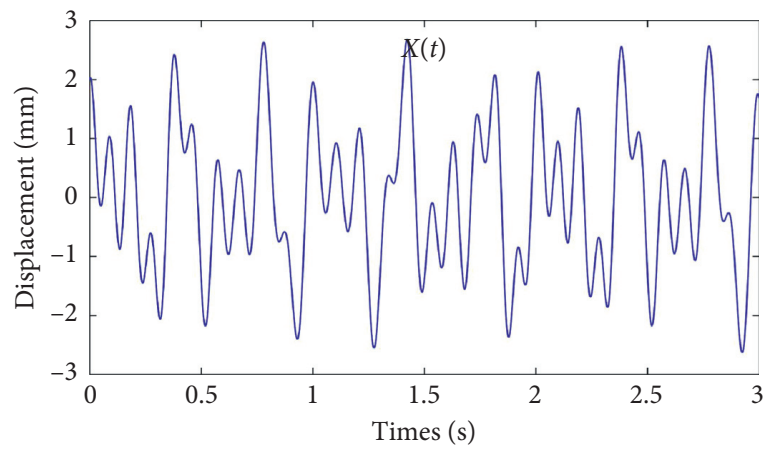

(d)

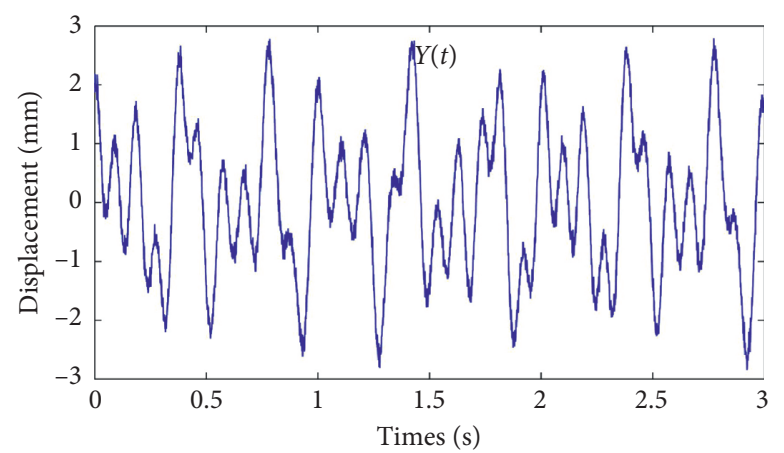

(e)

Figure 2: The waveforms of the simulated signal. (a) Waveform of the basic function $X_{1}$, (b) waveform of the basic function $X_{2}$, (c) waveform of the basic function of $X_{3}$, (d) waveform of the simulation signal $X(t)$, and (e) waveform of the simulation signal $Y(t)$.

\section{On-Site Experiment and Analysis}

4.1. Site Description and Data Acquisition. In this study, to further validate the accuracy of the proposed MF-ESMD denoising method for the obtained dynamic deflection by ground-based microwave interferometry, the Fengbei Bridge was selected as an experimental bridge. The Fengbei Bridge is one of the most important transportation hubs in Beijing, and it is located in the West Fourth Ring of Beijing, China, as shown in Figure 6(a). Moreover, there is a road crossing under the bridge, as shown in Figure 6(a). An Imaging by Interferometric Survey (IBIS-S) instrument, a typical system based on microwave interferometry, was located on one side of the bridge without passive radar reflectors attached to the bridge, as shown in Figure 6(b). Therefore, the instrument inevitably generated noise in the collected bridge dynamic deflection due to the surrounding environment, ground-motion, complex traffic activities, artificial operation, and the equipment itself. The IBIS-S instrument consisted of a radar unit, a control personal computer, a power supply unit, and a tripod. In typical measurement conditions, the sampling rate is up to $200 \mathrm{~Hz}$, the maximal detection distance is up to $1 \mathrm{~km}$, the range resolution is up to $0.50 \mathrm{~m}$, and the displacement measurement accuracy is up to $0.01 \mathrm{~mm}$. In this study, the angle of the altitude of the radar unit was set to 30 so that the two antennas on the radar unit could be aligned to the midspan point of the central span of the bridge. The range resolution was $0.5 \mathrm{~m}$, the sampling frequency was $200 \mathrm{~Hz}$, and the duration of data acquisition was $60 \mathrm{~s}$. The dynamic deflection obtained for the Fengbei Bridge with IBIS-S is shown in Figure 7. 


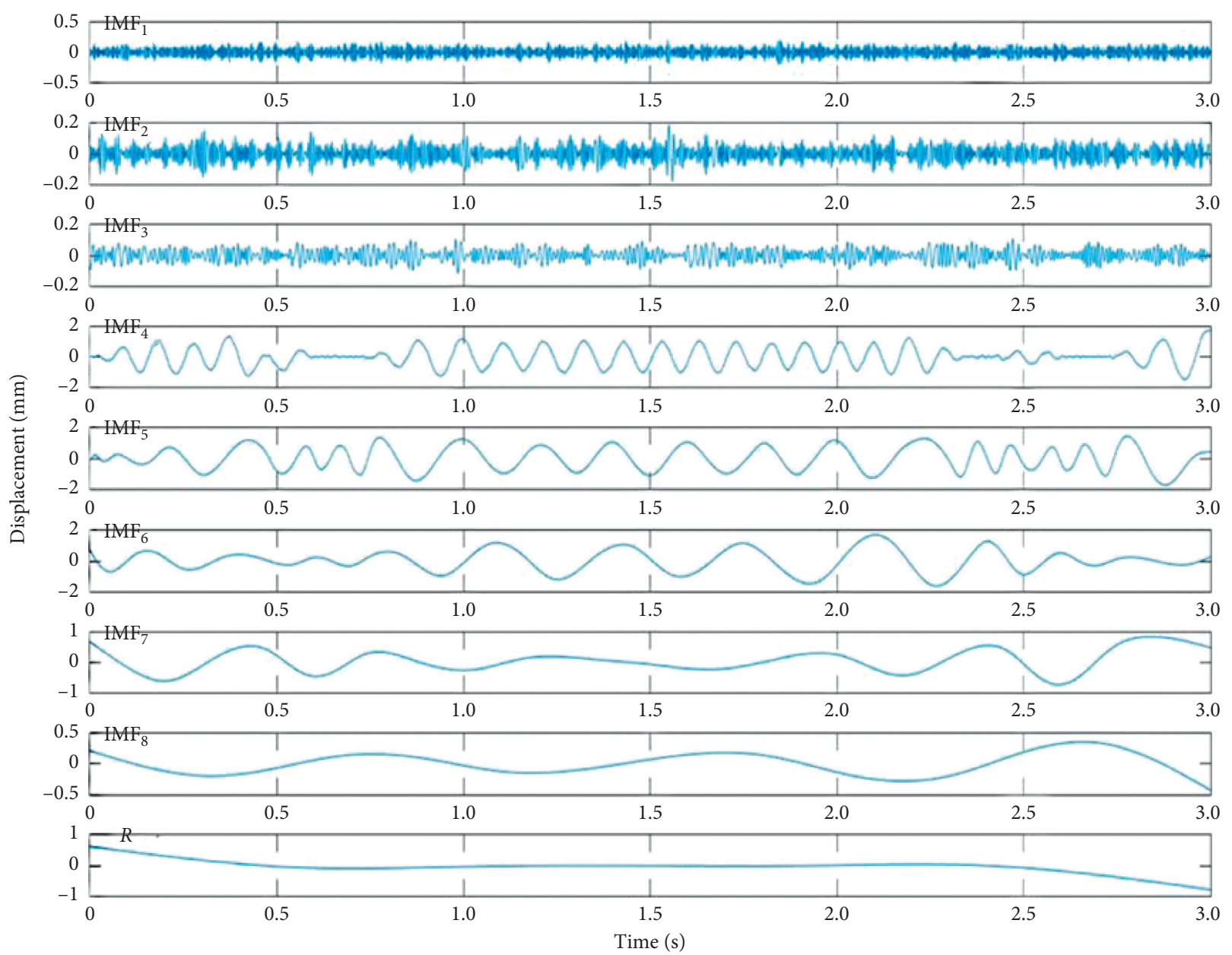

FIgURE 3: Decomposed IMFs of the simulation signal $Y(t)$ using the ESMD method.

TABLE 1: Spearman's rho values of IMFs and $R$.

\begin{tabular}{lcccccccr}
\hline $\mathrm{IMF}_{1}$ & $\mathrm{IMF}_{2}$ & $\mathrm{IMF}_{3}$ & $\mathrm{IMF}_{4}$ & $\mathrm{IMF}_{5}$ & $\mathrm{IMF}_{6}$ & $\mathrm{IMF}_{7}$ & $\mathrm{IMF}_{8}$ & $R$ \\
\hline 0.0505 & 0.0330 & 0.0291 & 0.5005 & 0.5644 & 0.5333 & 0.1554 & 0.0570 & 0.0706 \\
\hline
\end{tabular}

4.2. Result Analysis and Discussion. The original signal, the dynamic deflection of the Fengbei Bridge with IBIS-S, was first decomposed into 9 IMFs and a residual $R$ using the ESMD method, as shown in Figure 8. As described in Section 3, the simulated signal was decomposed into 8 IMFs, which was less than the number of the decomposed IMFs of the dynamic deflection of the Fengbei Bridge with IBIS-S. The reason is that the dynamic deflection signal is more complicated than the simulated signal, due to the influences of surrounding environment, wind thrust, ground motion, complex traffic activities, human operations, and the equipment itself. Then, Spearman's rho of each decomposed IMF was calculated, as shown in Table 3. According to Spearman's rho value, Spearman's rho of $\mathrm{IMF}_{3}$ was 0.0380 , which was the minimum value for the first time. Therefore, the first three IMF components, $\mathrm{IMF}_{1}$ to $\mathrm{IMF}_{3}$, were regarded as the noisedominant IMFs, which may have been caused by the surrounding environment, complex traffic activities, artificial operation, and the equipment itself. Then, the remaining IMF components were regarded as useful subsignals to be reconstructed as a new signal along with the residual $R$.

For the reconstructed signal, a mathematical morphology filter was used to perform the reduction of the detailed noise with the parameters $g_{1}=\left[\begin{array}{lll}1 & 1 & 1\end{array}\right]$ and $g_{2}=\left[\begin{array}{lllll}1 & 1 & 1 & 1 & 1\end{array}\right]$ in equation (6). The resulting denoised signals using the MFESMD method and the original signal are shown in Figure 9(a). Figures 9(b) and 9(c) show the comparison results between the original signal and the denoised signal using the morphological filter and the MF-EEMD methods [27, 29]. The inspection of the curves from this figure highlighted the following aspects: (1) compared with the original signal (the blue curve in Figure 9(a)), the denoised signal (the red curve in Figure 9(a)) using the MF-ESMD method was smoother and more stable, which indicated that the obvious noise information of the original signal was eliminated very well. (2) The denoised signal obtained by the MF-ESMD method was 


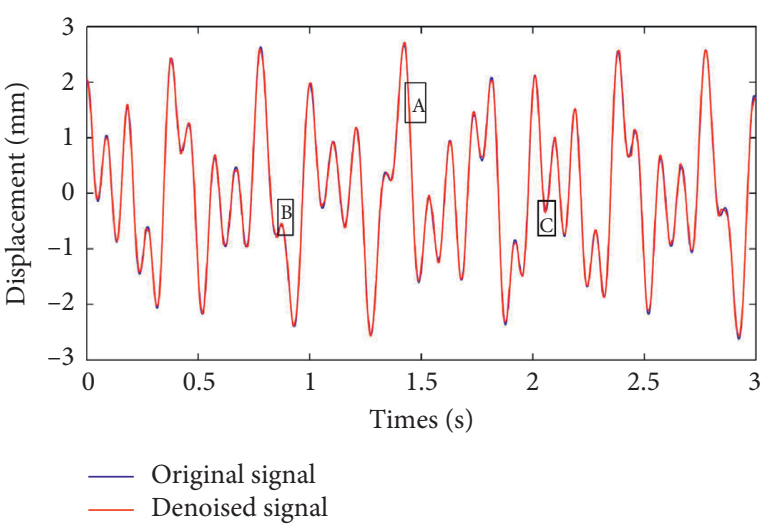

(a)

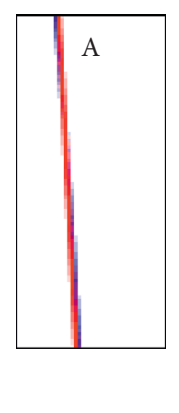

(b)

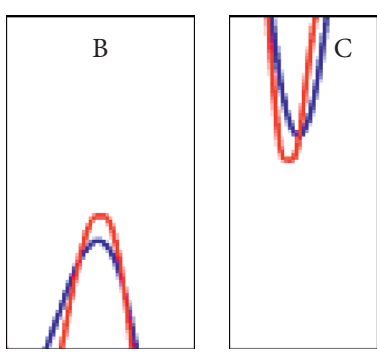

(c)

(d)

FIgURE 4: Curve comparison between $S(t)$ and $X(t)$. (a) Curve comparison between the original signal $X(t)$ and the denoised signal using the MF-ESMD method, (b) enlarged figure of area A in (a), (c) enlarged figure of area B in (a), and (d) enlarged figure of area C in (a).

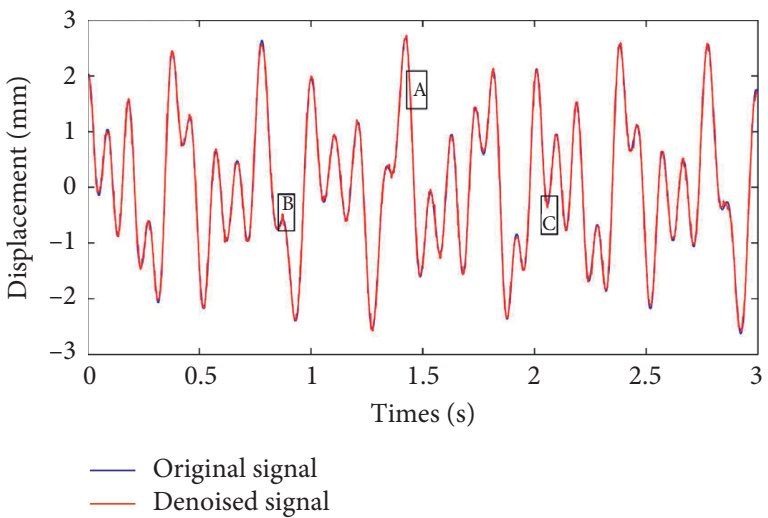

(a)

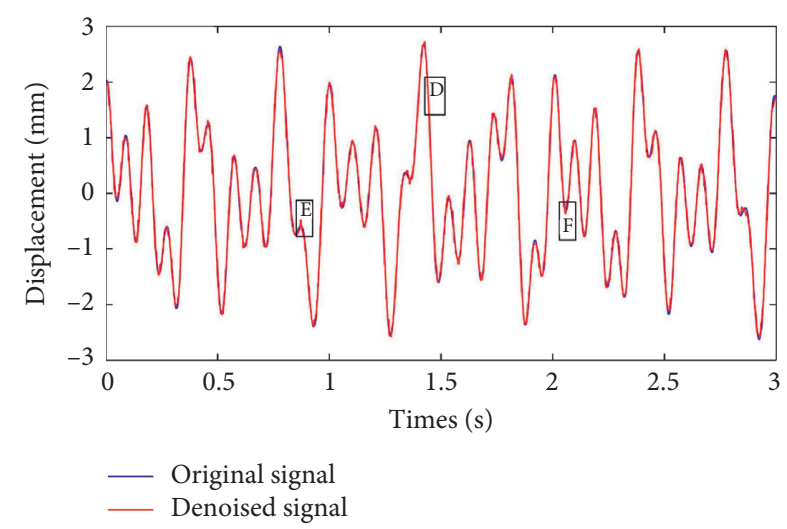

(e)
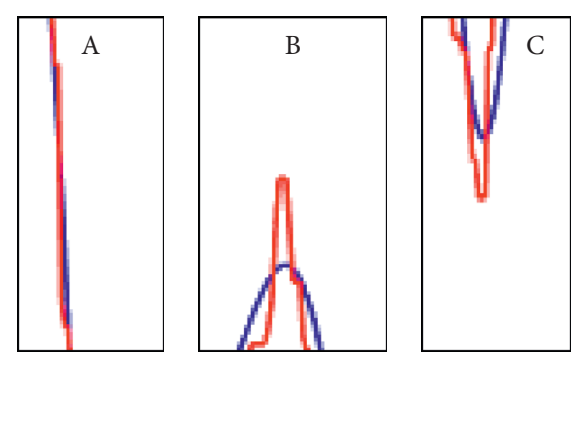

(b)

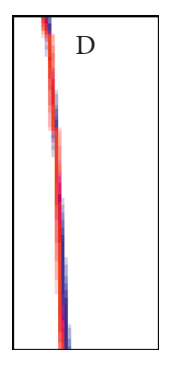

(f) (c)

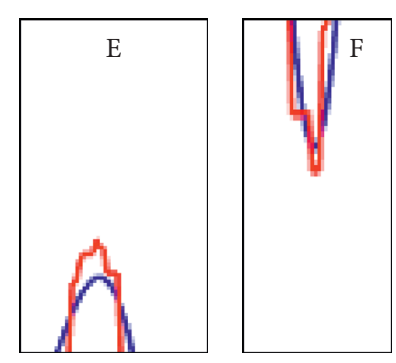

(g)

(h)

FIgURE 5: Curve comparison between the original signal $X(t)$ and the denoised signal using the morphological filter and MF-EEMD methods. (a) Curve comparison between the original signal $X(t)$ and the denoised signal using the morphological filter, (b) enlarged figure of area A in (a), (c) enlarged figure of area B in (a), (d) enlarged figure of area C in (a), (e) curve comparison between the original signal $X(t)$ and the denoised signal using the MF-EEMD method, (f) enlarged figure of area D in (e), (g) enlarged figure of area E in (e), and (h) enlarged figure of area $\mathrm{F}$ in (e).

smoother at the peaks and troughs of the curve than the denoised signal obtained by the morphological filter method and the MF-EEMD method. This indicated that the proposed MF-ESMD method had a better ability for the noise reduction in the peaks and troughs of the signal than the morphological filter method and the MF-EEMD method.

In this study, due to the complicated affecting factors, there were many kinds of noise in the obtained time series 
TABLE 2: The SNR and RMSE values of the denoised signal using the three denoising methods.

\begin{tabular}{lcc}
\hline & SNR (dB) & RMSE (mm) \\
\hline Morphological filter & 26.3066 & 0.0595 \\
MF-EEMD & 26.4298 & 0.0587 \\
MF-ESMD & 30.6619 & 0.0361 \\
\hline
\end{tabular}

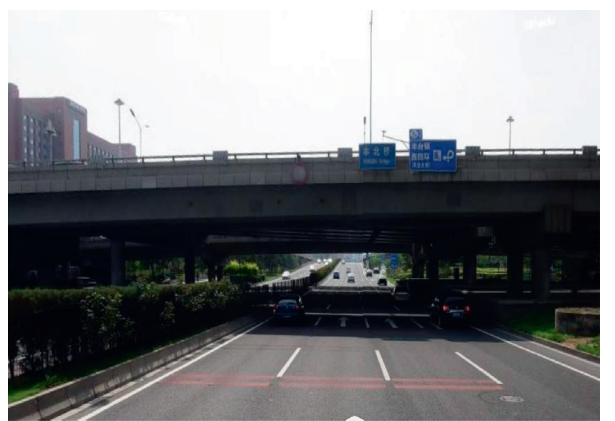

(a)

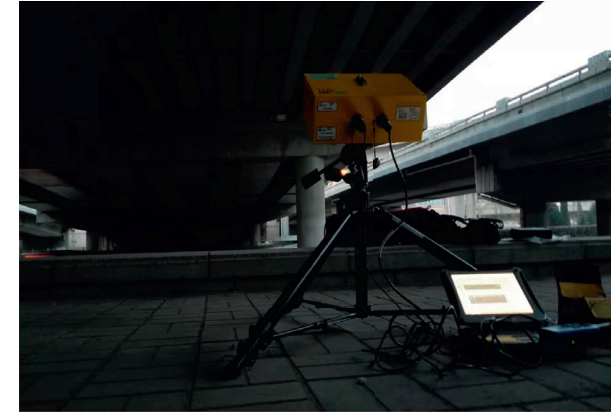

(b)

Figure 6: Fengbei Bridge and IBIS-S instrument layout. (a) Fengbei Bridge and (b) the dynamic deflection measurement of the Fengbei Bridge by IBIS-S.

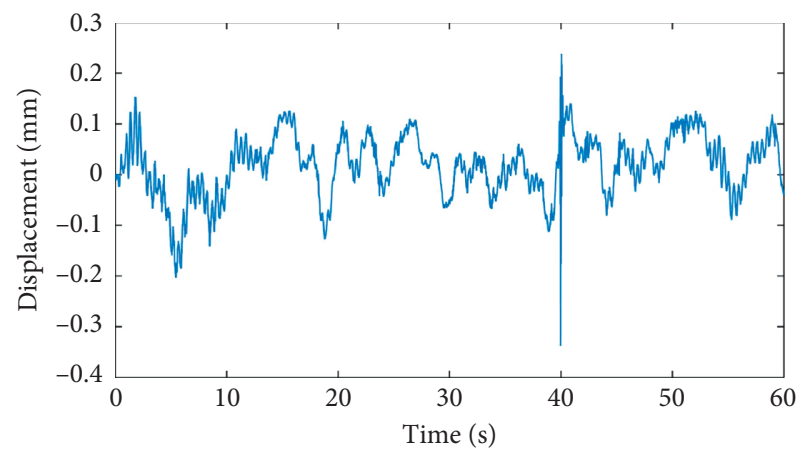

FIgURE 7: The waveform of the dynamic deflection of the Fengbei Bridge by IBIS-S.

displacement. It was difficult to evaluate the quality of signal denoising using the indexes of SNR and RMSE. Therefore, two new evaluation indexes, RVR and NRR, were used to evaluate the quality of signal denoising. The RVR was the ratio of the variance root of the difference number of the signal after denoising to the variance root of the difference of the original signal, which could reflect the smoothness of the noise signal [7]. The smaller the RVR, the better the denoised signal. The NRR could reflect the ability to suppress interference and improve the SNR [20]. In contrast, the bigger the NRR, the better the denoised signal:

$$
\begin{aligned}
& \operatorname{RVR}=\frac{\sum_{n=1}^{N-1}(\widetilde{s}(n+1)-\widetilde{s}(n))^{2}}{\sum_{n=1}^{N-1}(s(n+1)-s(n))^{2}}, \\
& \mathrm{NRR}=10\left(\lg \sigma_{1}^{2}-\lg \sigma_{2}^{2}\right),
\end{aligned}
$$

where $s(n)$ is the $n_{\text {th }}$ element of the original signal, $\widetilde{s}(n)$ is the $n_{\text {th }}$ element of the denoised signal, $N$ is the number of elements of the signal, $\sigma_{1}^{2}$ represents the variance of the measured original signal, and $\sigma_{2}^{2}$ denotes the variance of the denoised signals.

Table 4 shows the values of RVR and NRR for the denoised signals obtained by the morphological filter, the MF-EEMD method, and the proposed MF-ESMD method. The inspection from this table highlighted the following aspects: (1) the RVR was 0.0524 for the proposed MF-ESMD denoising method, which was smaller and closer to zero than the values for the morphological filter method and the MFEEMD method. (2) The NRR was 3.522 for the proposed MF-ESMD denoising method, which was better than the values of the morphological filter method and the MFEEMD method. However, the NRR was -4.9465 for the morphological filter method, which was a negative number. As shown in Figure 9(b), when the sampling time was approximately $40 \mathrm{~s}$, there was strong noise in the original signal, which blurred the characteristics of the useful signal using the mathematical morphology. Therefore, the morphological filter method could not distinguish the useful signal and noise, and it might treat the useful subsignals as the noise for the instant stronger noise. For the MF-EEMD method and the proposed MF-ESMD method, most of the obvious noises were reduced when the useful subsignal was reconstructed. There was only a small amount of detailed noise in the reconstructed signal, and the mathematical morphology filter was used to perform a further reduction of the detailed noise in the reconstructed signal. The results showed that the proposed MF-ESMD method had a good denoising ability, which not only efficiently reduced the 


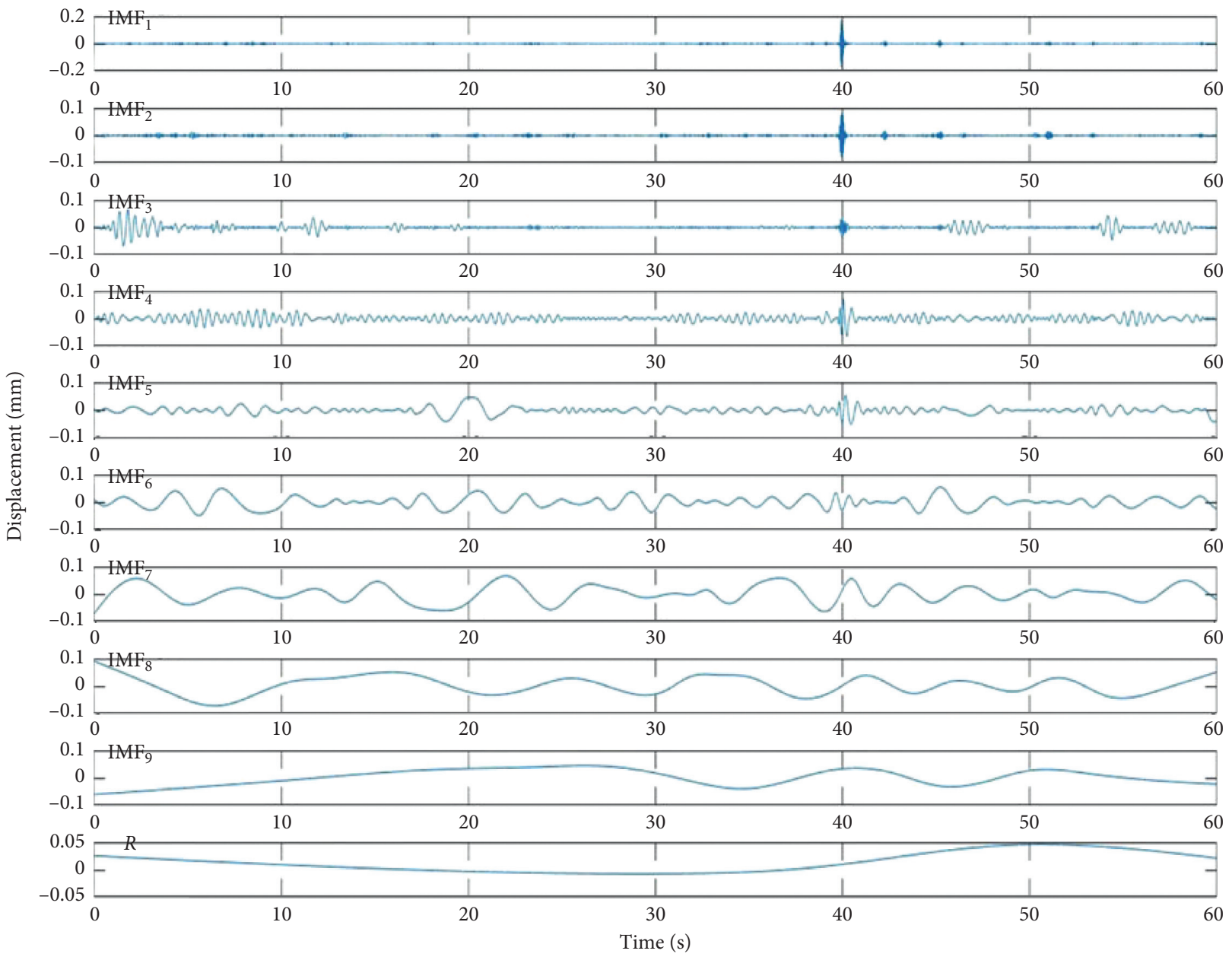

FIgURE 8: Decomposed IMFs of the original signal using the ESMD method.

TABle 3: Spearman's rho of the IMFs and $R$.

\begin{tabular}{lccccccccc}
\hline $\mathrm{IMF}_{1}$ & $\mathrm{IMF}_{2}$ & $\mathrm{IMF}_{3}$ & $\mathrm{IMF}_{4}$ & $\mathrm{IMF}_{5}$ & $\mathrm{IMF}_{6}$ & $\mathrm{IMF}_{7}$ & $\mathrm{IMF}_{8}$ & $\mathrm{IMF}_{9}$ & $R$ \\
\hline 0.0383 & 0.0380 & 0.1423 & 0.1522 & 0.2459 & 0.3558 & 0.4759 & 0.4149 & 0.3704 & 0.1148 \\
\hline
\end{tabular}

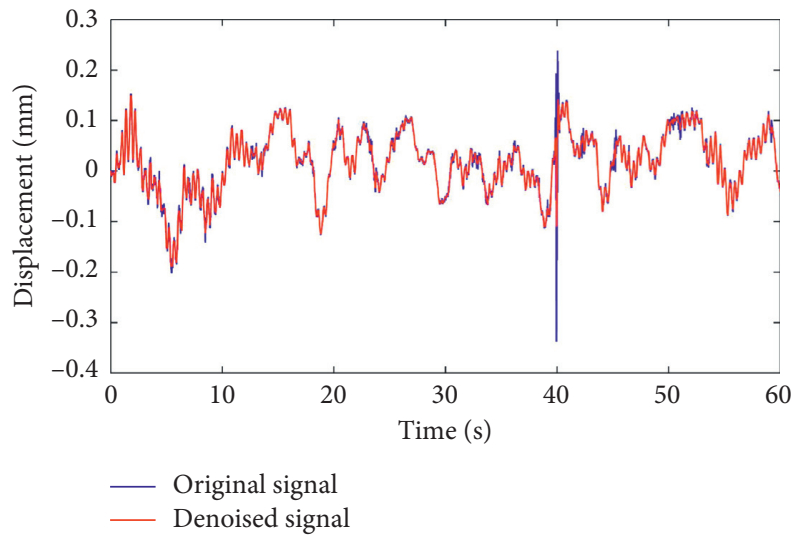

(a)

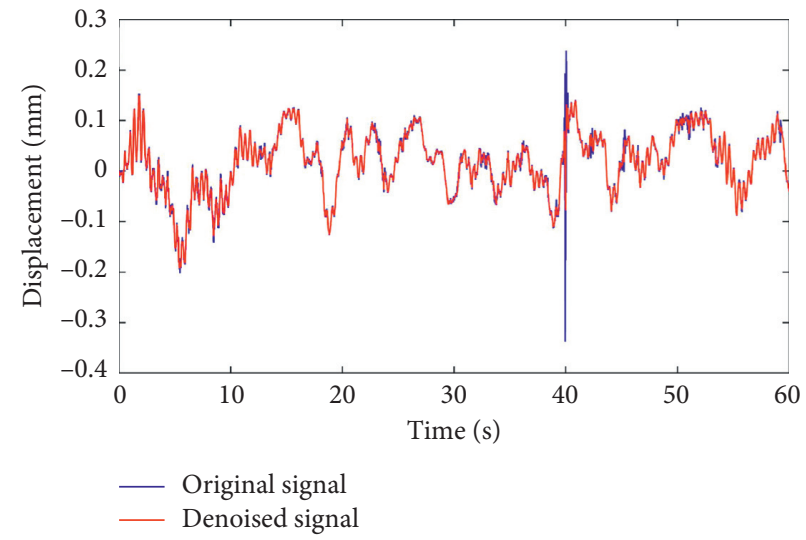

(b)

Figure 9: Continued. 


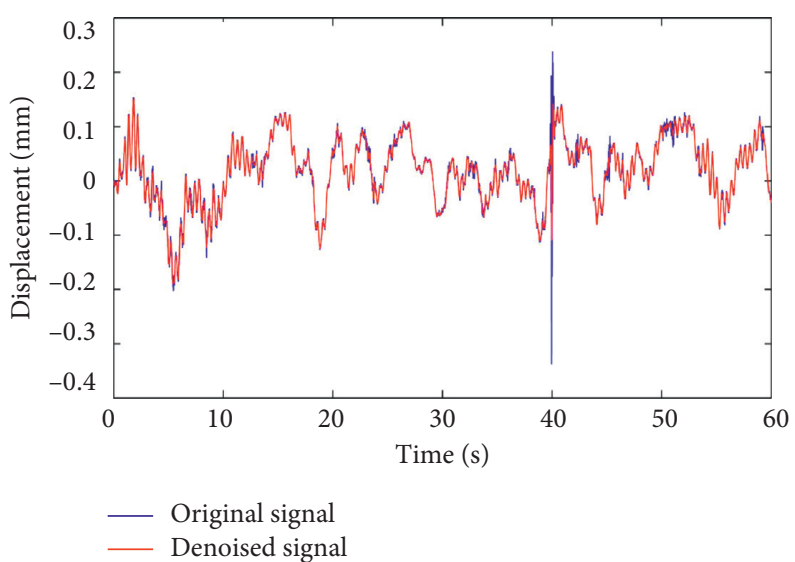

(c)

Figure 9: Curve comparison between the original signal and the denoised signal. (a) Curve comparison between the original signal and the denoised signal using the MF-ESMD methods; (b) curve comparison between the original signal and the denoised signal using the morphological filter; and (c) curve comparison between the original signal and the denoised signal using the MF-EEMD methods.

TABLE 4: The respective RVR and NRR values of the three methods.

\begin{tabular}{lcc}
\hline & RVR & NRR \\
\hline Morphological filter & 0.1361 & -4.9465 \\
MF-EEMD & 0.1650 & 1.5240 \\
MF-ESMD & 0.0524 & 3.5220 \\
\hline
\end{tabular}

effects of noise for dynamic deflection signals but could also retain useful information.

\section{Conclusions}

In recent years, ground-based microwave interferometry has been widely used to obtain the dynamic deflection measurements of bridges. Due to the influences of the surrounding environment and the equipment itself, there has been an inevitable increase in the noise information of the obtained dynamic deflection displacement. In this study, in order to improve the accuracy of the bridge dynamic deflection signals obtained by ground-based microwave interferometry, the MF-ESMD denoising method was proposed in order to remove the noise in the original signal. Through simulated experiments and real data experiments, it was found that the proposed method had the following advantages.

(1) The simulated experiment was performed in order to validate the feasibility and accuracy of the proposed MF-ESMD denoising method for a nonlinear and nonstationary simulated signal. The RMSE was $0.0361 \mathrm{~mm}$ and the SNR was $30.6619 \mathrm{~dB}$ for the proposed MF-ESMD denoising method. These values were much better than those of the morphological filter method and the MF-EEMD method. Moreover, the SNR of the proposed MF-ESMD denoising method increased by $53.3 \%$ compared with the SNR of the simulation signal. The results showed that the proposed MF-ESMD denoising method was useful for the signal denoising of the nonlinear and nonstationary signals.

(2) Compared with the morphological filter method and the MF-EEMD method, the MF-ESMD denoising method had better values for the indexes of RVR and NRR for the dynamic deflection displacement obtained using ground-based microwave interferometry. The results verified the feasibility and accuracy of the proposed MF-ESMD method for dynamic deflection displacement obtained using groundbased microwave interferometry.

(3) This MF-ESMD method integrated the ESMD method and Spearman's rho to remove the noisedominant IMFs from the decomposed IMFs for the bridge. A morphology filter was used to perform the reduction of the detailed noise in the reconstructed signal. Compared with the morphological filter method and the MF-EEMD method, the proposed MF-ESMD method had a good denoising ability, which not only efficiently reduced the effects of noise for the dynamic deflection signals but could also retain the useful information.

\section{Data Availability}

All the data included in this study are available upon request by contact with the corresponding author.

\section{Conflicts of Interest}

The authors declare no conflicts of interest.

\section{Authors' Contributions}

Xianglei Liu and Mengzhuo Jiang conceptualized the study. Mengzhuo Jiang and Ziqi Liu developed methodology. Mengzhuo Jiang and Hui Wang performed formal analysis. Ziqi Liu and Hui Wang were involved in data curation. 
Xianglei Liu performed English editing. Xianglei Liu was responsible for financial support.

\section{Acknowledgments}

This study was sponsored by the National Natural Science Foundation of China (grant no. 41871367), the Ministry of Science and Technology of the People's Republic of China (grant no. 2018YFE0206100), the Importation and Development of High-Caliber Talents Project of Beijing Municipal Institutions (grant no. CIT\&TCD201704053), the Science and Technology Project of Ministry of Housing and UrbanRural Development of the People's Republic of China (grant no. 2017-K4-002), the Scientific Research Project of Beijing Educational Committee (grant no. KM201910016007), the Major Projects of Beijing Advanced Innovation Center for Future Urban Design (grant no. UDC2018031321), and the BUCEA Post Graduate Innovation Project.

\section{References}

[1] F. Magalhães, A. Cunha, and E. Caetano, "Vibration based structural health monitoring of an arch bridge: from automated OMA to damage detection," Mechanical Systems and Signal Processing, vol. 28, pp. 212-228, 2012.

[2] G. W. Roberts, X. Meng, and A. H. Dodson, "Integrating a global positioning system and accelerometers to monitor the deflection of bridges," Journal of Surveying Engineering, vol. 130, no. 2, pp. 65-72, 2004.

[3] S. Yoneyama and H. Ueda, "Bridge deflection measurement using digital image correlation with camera movement correction," Materials Transactions, vol. 53, no. 2, pp. 285-290, 2012.

[4] R. Jiang and D. V. Jauregui, "Development of a digital closerange photogrammetric bridge deflection measurement system," Measurement, vol. 43, no. 10, pp. 1431-1438, 2010.

[5] L. Tian and B. Pan, "Remote bridge deflection measurement using an advanced video deflectometer and actively illuminated LED targets," Sensors, vol. 16, no. 9, p. 1344, 2016.

[6] G. Zhang, G. Guo, C. Yu, and L. Li, "Monitoring dynamic global deflection of a bridge by monocular digital photography," Stavebni Obzor-Civil Engineering Journal, vol. 27, no. 2, pp. 168-182, 2018.

[7] X. Liu, S. Li, and X. Tong, "Two-level W-ESMD denoising for dynamic deflection measurement of railway bridges by microwave interferometry," IEEE Journal of Selected Topics in Applied Earth Observations and Remote Sensing, vol. 11, no. 12, pp. 4874-4883, 2018.

[8] T. A. Stabile, A. Perrone, M. R. Gallipoli, R. Ditommaso, and F. C. Ponzo, "Dynamic survey of the Musmeci bridge by joint application of ground-based microwave radar interferometry and ambient noise standard spectral ratio techniques," IEEE Geoscience and Remote Sensing Letters, vol. 10, no. 4, pp. 870-874, 2013.

[9] C. Negulescu, G. Luzi, M. Crosetto et al., "Comparison of seismometer and radar measurements for the modal identification of civil engineering structures," Engineering Structures, vol. 51, pp. 10-22, 2013.

[10] X. Liu, Z. Lu, W. Yang, M. Huang, and X. Tong, "Dynamic monitoring and vibration analysis of ancient bridges by ground-based microwave interferometry and the ESMD method," Remote Sensing, vol. 10, no. 5, p. 770, 2018.
[11] M. Maizuar, L. Zhang, S. Miramini, P. Mendis, and R. G. Thompson, "Detecting structural damage to bridge girders using radar interferometry and computational modelling," Structural Control and Health Monitoring, vol. 24, no. 10, p. e1985, 2017.

[12] L. Zhou, J. Guo, J. Hu, J. Ma, F. Wei, and X. Xue, "Subsidence analysis of ELH bridge through ground-based interferometric radar during the crossing of a subway shield tunnel underneath the bridge," International Journal of Remote Sensing, vol. 39, no. 6, pp. 1911-1928, 2018.

[13] M. Pieraccini, M. Fratini, F. Parrini, and C. Atzeni, "Dynamic monitoring of bridges using a high-speed coherent radar," IEEE Transactions on Geoscience and Remote Sensing, vol. 44, no. 11, pp. 3284-3288, 2006.

[14] M. Alfaouri and K. Daqrouq, "ECG signal denoising by wavelet transform thresholding," American Journal of Applied Sciences, vol. 5, no. 3, pp. 276-281, 2008.

[15] J. Chen, J. Pan, Z. Li, Y. Zi, and X. Chen, "Generator bearing fault diagnosis for wind turbine via empirical wavelet transform using measured vibration signals," Renewable Energy, vol. 89, pp. 80-92, 2016.

[16] H. Sun, Y. Zi, Z. He, J. Yuan, X. Wang, and L. Chen, "Customized multiwavelets for planetary gearbox fault detection based on vibration sensor signals," Sensors, vol. 13, no. 1, pp. 1183-1209, 2013.

[17] L. Zhen, H. Zhengjia, Z. Yanyang, and W. Yanxue, "Customized wavelet de-noising using intra-and inter-scale dependency for bearing fault detection," Journal of Sound and Vibration, vol. 313, no. 1-2, pp. 342-359, 2008.

[18] S. K. Yadav, P. K. Bora, and R. Sinha, "Electrocardiogram signal denoising using non-local wavelet transform domain filtering," IET Signal Processing, vol. 9, no. 1, pp. 88-96, 2015.

[19] C. Liu, C. Song, and Q. Lu, "Random noise de-noising and direct wave eliminating based on SVD method for ground penetrating radar signals," Journal of Applied Geophysics, vol. 144, pp. 125-133, 2017.

[20] X. Liu, H. Wang, and M. Huang, "An improved second-order blind identification (SOBI) signal de-noising method for dynamic deflection measurements of bridges using groundbased synthetic aperture radar (GBSAR)," Applied Sciences, vol. 9, no. 17, p. 3561, 2019.

[21] X. Zhang, Y. Liang, J. Zhou, and Y. zang, "A novel bearing fault diagnosis model integrated permutation entropy, ensemble empirical mode decomposition and optimized SVM," Measurement, vol. 69, pp. 164-179, 2015.

[22] G. Han, B. Lin, and Z. Xu, "Electrocardiogram signal denoising based on empirical mode decomposition technique: an overview," Journal of Instrumentation, vol. 12, no. 3, Article ID P03010, 2017.

[23] J. B. Ali, N. Fnaiech, L. Saidi, B. Chebel-Morello, and F. Fnaiech, "Application of empirical mode decomposition and artificial neural network for automatic bearing fault diagnosis based on vibration signals," Applied Acoustics, vol. 89, pp. 16-27, 2015.

[24] A. R. Hassan and M. I. H. Bhuiyan, "Automated identification of sleep states from EEG signals by means of ensemble empirical mode decomposition and random under sampling boosting," Computer Methods and Programs in Biomedicine, vol. 140, pp. 201-210, 2017.

[25] A. Tabrizi, L. Garibaldi, A. Fasana, and S. Marchesiello, "Early damage detection of roller bearings using wavelet packet decomposition, ensemble empirical mode decomposition and support vector machine," Meccanica, vol. 50, no. 3, pp. 865-874, 2015. 
[26] X.-H. Chen, G. Cheng, X.-L. Shan, X. Hu, Q. Guo, and H.-G. Liu, "Research of weak fault feature information extraction of planetary gear based on ensemble empirical mode decomposition and adaptive stochastic resonance," Measurement, vol. 73, pp. 55-67, 2015.

[27] J. Chan, H. Ma, T. Saha, and C. Ekanayake, "Self-adaptive partial discharge signal de-noising based on ensemble empirical mode decomposition and automatic morphological thresholding," IEEE Transactions on Dielectrics and Electrical Insulation, vol. 21, no. 1, pp. 294-303, 2014.

[28] S. Wang, N. Zhang, L. Wu, and Y. Wang, "Wind speed forecasting based on the hybrid ensemble empirical mode decomposition and GA-BP neural network method," Renewable Energy, vol. 94, pp. 629-636, 2016.

[29] X. Zhou, D. Shan, and Q. Li, "Morphological filter-assisted ensemble empirical mode decomposition," Mathematical Problems in Engineering, vol. 2018, Article ID 5976589, 12 pages, 2018.

[30] J. Li, Y. Tong, L. Guan, S. Wu, and D. Li, "A UV-visible absorption spectrum denoising method based on EEMD and an improved universal threshold filter," RSC Advances, vol. 8, no. 16, pp. 8558-8568, 2018.

[31] H. Chen, P. Chen, W. Chen, C. Wu, J. Li, and J. Wu, "Wind turbine gearbox fault diagnosis based on improved EEMD and Hilbert square demodulation," Applied Sciences, vol. 7, no. 2, p. 128, 2017.

[32] J. L. Wang and Z. J. Li, "Extreme-point symmetric mode decomposition method for data analysis," Advances in Adaptive Data Analysis, vol. 5, no. 3, Article ID 1350015, 2013.

[33] X. Liu, Y. Tang, Z. Lu, H. Huang, X. Tong, and J. Ma, "ESMDbased stability analysis in the progressive collapse of a building model: a case study of a reinforced concrete frameshear wall model," Measurement, vol. 120, pp. 34-42, 2018.

[34] J. Wang and X. Fang, "ESMD method for frequency distribution of tank surface temperature under wind effect," International Journal of Geosciences, vol. 6, no. 5, pp. 481-486, 2015.

[35] J. R. Lei, Z. H. Liu, and L. Bai, "The regional features of precipitation variation trends over Sichuan in China by the ESMD method," Mausam, vol. 67, no. 11, pp. 849-860, 2016.

[36] A. Hu and L. Xiang, "Selection principle of mathematical morphological operators in vibration signal processing," Journal of Vibration and Control, vol. 22, no. 14, pp. 31573168, 2016.

[37] H. Li, R. Wang, S. Cao, Y. Chen, and W. Huang, "A method for low-frequency noise suppression based on mathematical morphology in microseismic monitoring," Geophysics, vol. 81, no. 3, pp. V159-V167, 2016.

[38] M. Van, P. Franciosa, and D. Ceglarek, "Rolling element bearing fault diagnosis using integrated nonlocal means denoising with modified morphology filter operators," Mathematical Problems in Engineering, vol. 2016, Article ID 9657285, 14 pages, 2016.

[39] Z. Hua, L. Junhao, and Q. D. Shi, “A preprocessing method for flutter signals based on morphological filtering," in Proceedings of the 9th International Conference on Mechanical And Aerospace Engineering (ICMAE), pp. 430-434, Budapest, Hungary, September 2018.

[40] J. Lv and J. Yu, "Average combination difference morphological filters for fault feature extraction of bearing," $M e$ chanical Systems and Signal Processing, vol. 100, pp. 827-845, 2018.

[41] Y. Li, X. Liang, W. Liu, and Y. Wang, "Development of a morphological convolution operator for bearing fault detection," Journal of Sound and Vibration, vol. 421, pp. 220-233, 2018.

[42] T. Bai, D. Li, H. Wang et al., "A PPG signal de-noising method based on the DTCWT and the morphological filtering," in Proceedings of the 12th International Conference on SignalImage Technology \& Internet-Based Systems (SITIS), pp. 503-506, Naples, Italy, December 2016.

[43] S. Mukhopadhyay and B. Chanda, "An edge preserving noise smoothing technique using multiscale morphology," Signal Processing, vol. 82, no. 4, pp. 527-544, 2001.

[44] W. Xu, Y. Hou, Y. S. Hung, and Y. Zou, "A comparative analysis of Spearman's rho and Kendall's tau in normal and contaminated normal models," Signal Processing, vol. 93, no. 1, pp. 261-276, 2013.

[45] K. Smeds, F. Wolters, and M. Rung, "Estimation of signal-tonoise ratios in realistic sound scenarios," Journal of the American Academy of Audiology, vol. 26, no. 2, pp. 183-196, 2015. 